\title{
Robôs Evolutivos para a Investigação do Comportamento de Ratos no Teste de Campo Aberto
}

\author{
Luciana T. Raineri ${ }^{1}$, Rafael S. Del Lama ${ }^{1}$, Raquel M. Candido ${ }^{1}$, \\ Ariadne Costa ${ }^{2}$, Renato Tinos ${ }^{1}$ \\ ${ }^{1}$ Departamento de Computação e Matemática, FFCLRP - Universidade de São Paulo (USP) \\ Ribeirão Preto - SP - Brasil \\ ${ }^{2}$ Universidade Acadêmica Especial de Ciências Exatas - Universidade Federal de Goiás \\ Jataí - GO - Brasil \\ \{luciana.traineri,ad.andrade.costa\}@gmail.com, rtinos@ffclrp.usp.br
}

\begin{abstract}
The open field test is a widely used experiment to test levels of motor activity and anxiety in rodents, allowing the study of anxiolytic or anxiogenic pharmacological compounds. In this work, a computational model was developed for virtual rats based on evolutionary robots for the open field test. The virtual rat is controlled by a recurrent neural network optimized by genetic algorithms (GAs). The fitness function used in the GA simulates the conflict between fear and exploratory behavior of the rat in the open field. Simulations and experiments with a real robot indicate that the evolved virtual rat and real rats present similar bahavior in the open field test.
\end{abstract}

\begin{abstract}
Resumo. O teste do campo aberto é um experimento bastante utilizado para teste de níveis de atividade motora e ansiedade em roedores, permitindo o estudo de compostos farmacológicos ansiolíticos ou ansiogênicos. Neste trabalho, foi desenvolvido um modelo computacional para ratos virtuais baseados em robôs evolutivos para o teste de campo aberto. O rato virtual é controlado por uma rede neural recorrente otimizada por algoritmos genéticos (AGs). A função de fitness utilizada no AG corresponde ao conflito entre medo e caráter exploratório do rato no campo aberto. Simulações e um experimento com o robô real indicam que o rato virtual otimizado tem comportamentos similares aos de ratos reais no teste do campo aberto.
\end{abstract}

\section{Introdução}

Para tentar responder questões sobre mecanismos de comportamento, modelos animais são amplamente utilizados por pesquisadores. O Teste de Campo Aberto [Walsh and Cummins 1976] é um dos experimentos mais utilizados em Psicobiologia para medir comportamentos em modelos animais, principalmente em modelos de roedores [Seibenhener and Wooten 2015]. Trata-se de um teste rápido em que o rato explora uma arena retangular livre de obstáculos. Tal modelo fornece uma variedade de informações comportamentais, permitindo o teste de níveis de atividade motora e o estudo de diferentes compostos farmacológicos para efeitos ansiolíticos ou ansiogênicos.

Outro experimento de grande valia para estudos neurobiológicos é o labirinto em cruz elevado (LCE), que consiste em dois braços retangulares, ortogonais e com ponto 
central coincidente [Hogg 1996]. Apenas um dos braços é aberto, enquanto o outro é circundado por paredes (braço fechado) [Giddings 2002].

Os experimentos com animais são burocraticamente restritos e podem trazer sofrimento para os roedores, que muitas vezes são mortos após os experimentos. Nesse contexto, a utilização de modelos computacionais é uma alternativa válida para auxiliar o estudo de modelos comportamentais [Salum et al. 2000], [Miranda et al. 2009], [Tejada et al. 2010], [Costa 2015]. A criação de um modelo computacional para o rato permite também que os efeitos de drogas sejam testados por meio de simulações de um rato virtual, com comportamentos semelhantes ao do rato real. No contexto deste trabalho, o rato virtual é um agente computacional inteligente.

Em trabalhos prévios do nosso grupo de pesquisa, como [Shimo et al. 2010], [Costa et al. 2014] e [de Andrade Costa and Tinós 2016], foram propostos dois modelos para o rato virtual navegando no LCE. Em ambos, os ratos virtuais são controlados por redes neurais recorrentes, cujos pesos são otimizados por um algoritmo genético (AG). No primeiro modelo [Shimo et al. 2010], dados obtidos em experimentos com ratos reais são utilizados para o treinamento da rede neural que controla o rato virtual. No segundo modelo computacional [Costa 2015], utiliza-se um modelo comportamental baseado no conflito entre medo e exploração [Montgomery 1955] para o projeto do rato virtual, que neste caso é simulado. Dados dos ratos reais são utilizados apenas para validação do rato virtual. Apesar dos resultados interessantes em [Costa 2015], modelos virtuais de organismos biológicos podem sofrer uma séria restrição: as interações entre o ser vivo e o ambiente são, em geral, difíceis de serem simuladas devido ao enorme número de variáveis envolvidas. Como alternativa, robôs autônomos podem servir como uma importante ferramenta para o desenvolvimento e teste de modelos de comportamentos, de habilidades cognitivas e de modelos evolutivos de organismos vivos [Webb 2001].

Assim, este trabalho teve como objetivo principal propor uma metodologia para o desenvolvimento de ratos virtuais baseados em robôs evolutivos para o teste de campo aberto. Tendo como base o trabalho desenvolvido em [Costa 2015] para ratos virtuais no LCE, o agente artificial é controlado por uma rede neural recorrente com pesos otimizados por um AG. Outra contribuição deste trabalho é a utilização de redes neurais recorrentes do tipo Echo State Network (ESN) [Jaeger and Haas 2004] otimizadas por AGs para o controle do rato virtual.

\section{Metodologia}

Neste trabalho, os ratos virtuais baseados em robôs evolutivos para o teste de campo aberto são controlados por ESNs. As conexões recorrentes são necessárias para que o robô tenha comportamentos dinâmicos complexos, i.e., não simplesmente reativos. Devido ao tempo computacional longo para o experimento com o AG, os pesos da ESN foram obtidos em simulações. O indivíduo final foi então testado em um robô real. Foi utilizado um robô que tem as seguintes características: três motores de corrente contínua ligados a rodas omnidirecionais; cinco sensores infravermelhos colocados ao redor do robô de modo a permitir a detecção de obstáculos; um micro-controlador MSP 430; um rádio transmissor-receptor, utilizado para comunicação da placa-mãe do robô com um computador central.

Como aqui não é possível utilizar o aprendizado supervisionado para o treina- 
mento da rede (pois não se conhece a ação que o robô deve tomar em cada posição de sua trajetória), os vetores de pesos entre a camada intermediária (reservatório) e a camada de saída são otimizados por AGs. Na Figura 1 são mostrados como o AG é aplicado à otimização do vetor de pesos utilizando-se o robô móvel e a estrutura da ESN.

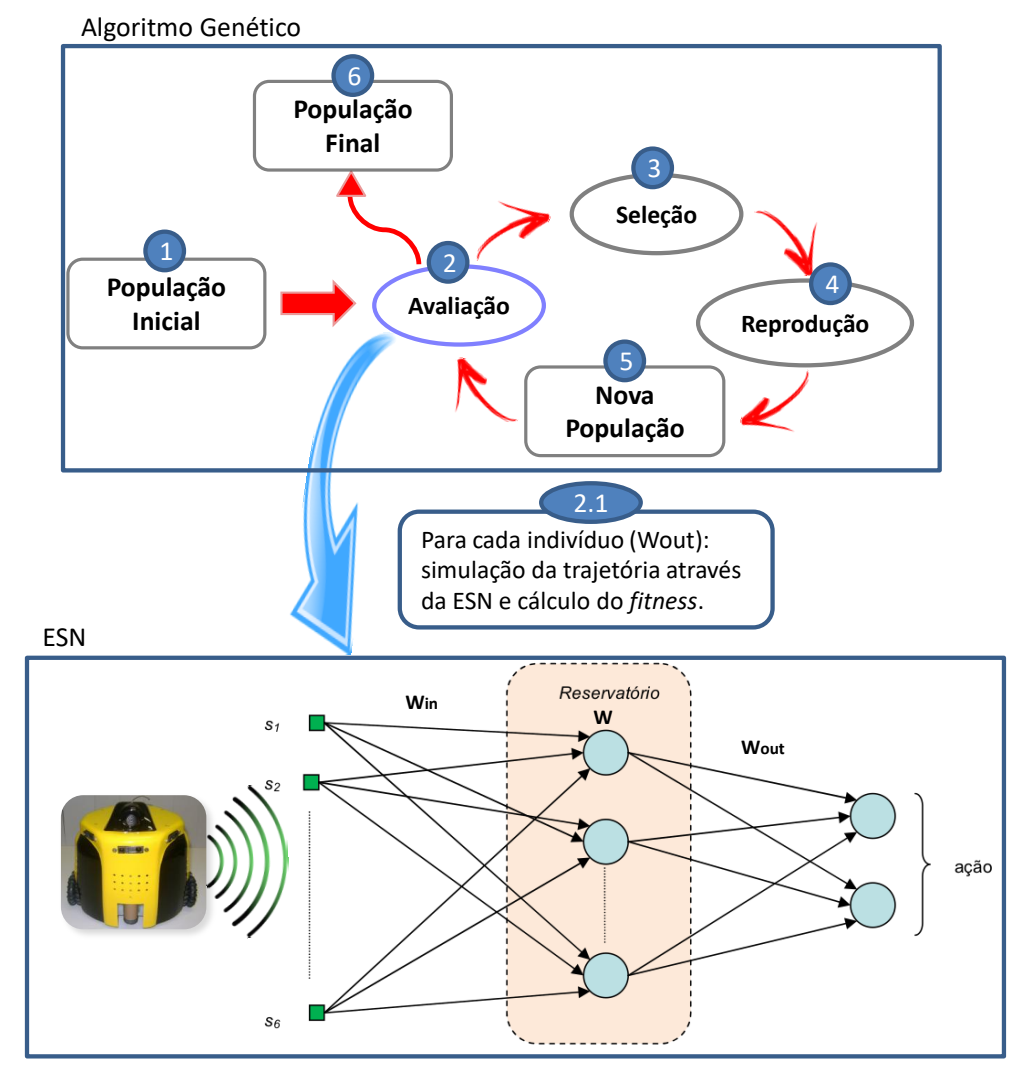

Figura 1. Algoritmo Genéico e RNA recorrente (Echo state Network - ESN) utilizada para controlar o robô. Por simplicidade, as conexões recorrentes dos neurônios localizados no reservatório não são mostradas.

Os parâmetros da rede foram definidos de acordo com as especificidades deste trabalho. Assim, o vetor de entrada tem tamanho seis, referente às seis leituras dos sensores de distância do robô $\left(s_{1}, \ldots, s_{6}\right)$. Esses sensores têm saída igual a 1 quando estão ativados, i.e., se existe um obstáculo a uma distância menor que o limiar $r$, e 0 caso contrário. As medidas $s_{1}, s_{3}$ e $s_{5}$ são para $r=10 \mathrm{~cm}$, enquanto que $s_{2}, s_{4}$ e $s_{6}$ são para $r=30 \mathrm{~cm}$.

Para cada posição da arena em que o robô estiver navegando, a ESN gera uma saída correspondente a uma ação, tendo como entrada seis leituras dos sensores de distância do robô $\left(s_{1}, \ldots, s_{6}\right)$. O número de ações que o robô pode tomar é quatro: andar para frente, ou girar $45^{\circ},-45^{\circ}$ ou $90^{\circ}$. Logo, o número de neurônios na camada de saída foi fixado em quatro nos experimentos realizados. Assim, o neurônio com máxima ativação define a ação tomada pela ESN em cada instante de tempo. O valor utilizado para o número de neurônios do reservatório nos experimentos foi definido empiricamente como 50.

Apenas os pesos entre a camada intermediária (reservatório) e a camada de saída são otimizados por um AG; os pesos do reservatório e os pesos entre a camada de entrada 
e o reservatório são fixos durante o treinamento. Eles são gerados aleatoriamente e então normalizados no início das execuções do AG. A recorrência entre os neurônios do reservatório é esparsa, sendo fixada uma taxa de 1\%, de acordo com [Jaeger and Haas 2004].

No AG, o indivíduo (vetor de pesos (x)) é avaliado após um determinado número de passos de tempo do rato virtual na arena. O fitness do indivíduo foi construído para modelar o comportamento do rato proposto por [Montgomery 1955]. Montgomery explica que o comportamento do rato em um ambiente é resultado do conflito entre medo e curiosidade. Neste trabalho, o fitness é calculado de acordo com a trajetória realizada pelo rato virtual, sendo composto por um somatório envolvendo dois termos: o primeiro termo é referente à recompensa para o caráter exploratório (curiosidade) do agente; já o segundo representa o perigo no qual o agente é exposto em diferentes regiões da arena (medo). A função de fitness é apresentada na Equação 1:

$$
f(\mathbf{x})=\sum_{t=1}^{n}\left(r\left(a_{t}, \mathbf{s}_{t}, \mathbf{m}_{t}\right)+\beta p\left(\mathbf{s}_{t}\right)\right)
$$

sendo $n$ o número de passos de tempo do rato virtual no campo aberto (iterações); $a_{t}$ a ação tomada pelo robô no instante de tempo $t ; \mathbf{s}_{t}$ a leitura dos sensores $\left(\mathbf{s}_{t 1}, \ldots, \mathbf{s}_{t 6}\right) ; \mathbf{m}_{t}$ o vetor onde são armazenadas as $b$ últimas leituras dos sensores; $b$ o tamanho do vetor de memória; $r\left(a_{t}, \mathbf{s}_{t}, \mathbf{m}_{t}\right)$ a recompensa, que é igual a 1 se a ação é andar para frente e $z<h\left(\mathbf{s}_{t}, \mathbf{m}_{t}\right)$, e 0 caso contrário; $z$ um número aleatório com distribuição uniforme no intervalo [0,1]; $h\left(\mathbf{s}_{t}, \mathbf{m}_{t}\right)$ a variável que assume valor 1 se a leitura atual dos sensores não se iguala à nenhuma das leituras armazenadas em $\mathbf{m}_{t}$ e $h\left(\mathbf{s}_{t}, \mathbf{m}_{t}\right)=1-\gamma / g\left(\mathbf{s}_{t}, m_{t}\right)$, caso contrário; $\gamma$ uma constante escolhida no intervalo [0,1]; $g\left(\mathbf{s}_{t}, \mathbf{m}_{t}\right)$ a posição no vetor onde $\mathbf{s}_{t}$ se iguala a $\mathbf{m}_{t}[i]$, para alguma posição $i ; p\left(\mathbf{s}_{t}\right)$ a punição. É igual a -1 se $l<k\left(\mathbf{s}_{t}\right)$, e 0 , caso contrário, sendo $l$ um número aleatório com distribuição uniforme no intervalo $[0,1] ; k\left(\mathbf{s}_{t}\right)$ a variável que pode assumir valores entre $\alpha$ e $\alpha / 7$, de acordo com a equação: $k\left(\mathbf{s}_{t}\right)=\alpha /(q+1)$, onde $q$ é o número de sensores ativados na leitura $\mathbf{s}_{t} ; \alpha$ uma constante escolhida no intervalo $[0,1] ; \beta$ o parâmetro que controla a importância relativa de cada um dos dois termos conflitantes da função de fitness. A função de fitness foi adaptada de [Costa 2015], onde o rato virtual foi desenvolvido para o LCE.

\section{Experimentos}

O simulador desenvolvido para o robô que navega em uma arena retangular foi utilizado na realização de experimentos. Na ESN, foram mantidos constantes os seguintes parâmetros: tamanho do vetor de entrada, 6 (número de sensores do robô); número de neurônios na camada de saída, 4 (número de ações do robô); número de neurônios no reservatório, 50; taxa de recorrência no reservatório, 1\%. No AG, os parâmetros mantidos constantes foram: número de passos de tempo do rato virtual (Equação 1), $n=300$. Esse valor foi escolhido pois equivale ao tempo (em segundos) em que, geralmente, os ratos são observados na arena [BAILEY \& CRAWLEY, 2009]; número de execuções, 30; número de gerações, 500; tamanho da população, 100 indivíduos; $\alpha=0,07 ; \gamma=0,9$.

Para simular o efeito de ansiedade no rato, foram escolhidos dois valores para $\beta$ : 0,5 e 6 . O primeiro, $\beta=0,5$, foi escolhido para simular comportamentos de ratos com menos ansiedade, que tendem a explorar mais o ambiente. O segundo, $\beta=6$, foi escolhido para aumentar o peso da punição (segundo termo da Equação 1) e simular com- 
portamentos de ratos mais ansiosos, onde o medo se torna um fator importante. Alterando o valor de $\beta$, espera-se que o modelo consiga reproduzir o comportamento de ratos sob o efeito de diferentes dosagens de drogas para controle de ansiedade. Em geral, os ratos evitam áreas mais expostas da arena, como a região central. Ambos os valores foram utilizados em experimentos com tamanho de memória $b=10$.

\section{Resultados}

Os resultados obtidos foram analisados e comparados considerando-se três regiões da arena da simulação, como mostrado na Figura 2. Do mesmo modo como é feito em experimentos com ratos reais no teste do campo aberto, foram contabilizadas as seguintes métricas para a análise das trajetórias dos ratos virtuais: tempo em cada região, número de entradas em cada região e distância percorrida na região central.

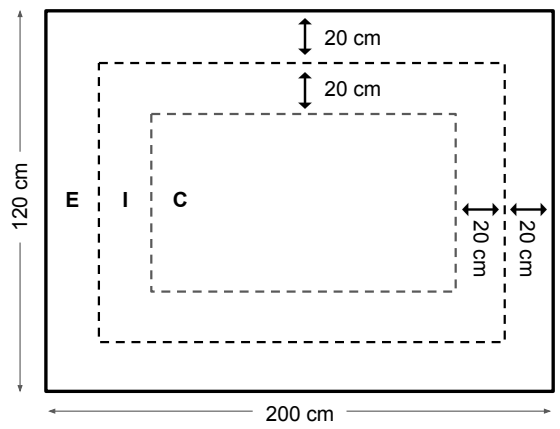

Figura 2. A arena da simulação $(200 \mathrm{~cm} \times 120 \mathrm{~cm})$ e as três regiões consideradas: 1) Região Externa (E), 2) Região Interna (I) e 3) Região Central (C).

Através das trajetórias obtidas nas simulações, foi possível observar que, para $\beta=$ 6 , o rato virtual tende a ficar mais tempo na região externa da arena. Para ambos os betas, ocorre um movimento repetido nos cantos da arena, que acaba sendo mais interessante para o rato. Isso ocorre porque as leituras dos sensores variam razoavelmente e, assim, a probabilidade de ganhar fitness aumenta. Além disso, a probabilidade de punição na região externa é menor em relação às outras regiões. Apesar disso, a punição por andar nas regiões intermediária e central é menor para $\beta=0,5$, o que possibilita o aparecimento de um comportamento mais exploratório, onde o rato percorre todas as regiões da arena.

Na Figura 3 são apresentados os resultados quantitativos para as simulações. Foram calculadas as médias de 30 execuções do $\mathrm{AG} \operatorname{com} \beta=0,5$ e $\beta=6$ para as métricas citadas acima: 1) Média do número de entradas do rato virtual paras as três regiões (Figura 3.a), 2) Média do tempo de permanência em cada uma das três regiões (Figura 3.b) e 3) Média da distância percorrida na região central (Figura 3.c). Utilizou-se o teste de Wilcoxon (signed ranks) e as diferenças entre os grupos foram consideradas estatisticamente significantes para o nível de significância $p<0,001$ e $p<0,01$.

Como discutido anteriormente, para $\beta=0,5$ o rato virtual possui mais de um tipo de comportamento e a média do número de entradas nas três regiões e a média da distância percorrida na região central têm grandes desvios (Figura 3.a e 3.c). Para $\beta=6$, o rato virtual tende a permanecer na região externa da arena, por isso, na Figura 3.b é possível ver que o tempo médio na região externa é maior do que o tempo médio nas 
(a)

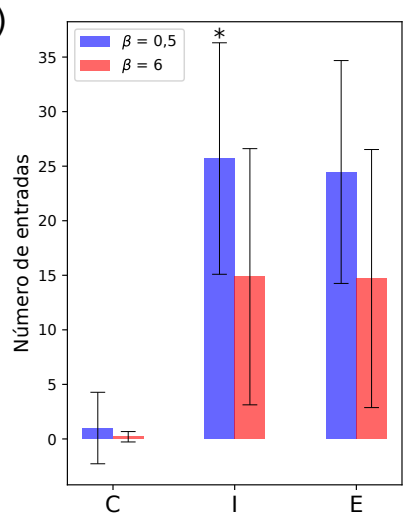

(b)

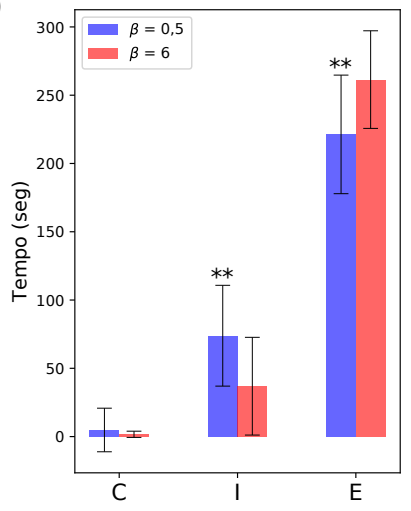

(c)

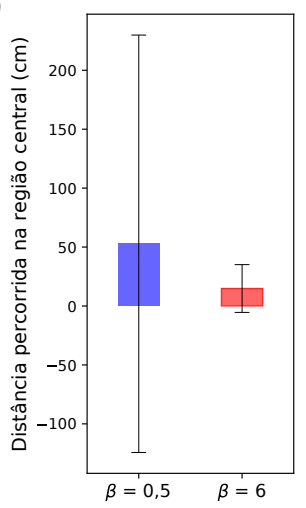

Figura 3. Resultados da simulação para o teste do campo aberto com memória 10 e $\beta=0,5$ (azul) e $\beta=6$ (vermelho). (a) Média do número de entradas do rato virtual para as três regiões (C, I, E), (b) Média do tempo de permanência em cada uma das três regiões (C, I, E) e (c) Média da distância percorrida na região central. Os símbolos $\left(^{*}\right)$ e $\left(^{* *}\right)$ indicam que os resultados para $\beta=0,5$ e $\beta=6$ são de populações diferentes de acordo com o teste de Wilcoxon (signed ranks) com $p<0,01$ e $p<0,001$, respectivamente.

outras regiões. Os ratos com $\beta=6$ também possuem mais de um tipo de comportamento, como permanecer mais nos cantos ou andar pelas regiões externa e intermediária da arena, por isso o desvio maior na média do número de entradas nessas regiões (Figura 3.a). De maneira geral, os ratos virtuais possuem comportamentos parecidos para ambos os valores de $\beta$, mas $\operatorname{com} \beta=0,5$ os ratos podem ter um comportamento de explorar a região central e intermediária que não acontece para $\beta=6$. Assim, as diferenças estatisticamente significantes entre eles ocorrem apenas para o número de entradas na região intermediária $(p<0,01)$ e tempo gasto nas regiões intermediária e externa $(p<0,001)$.

Para obter um comportamento mais uniforme para o rato virtual, a função de fitness da Equação 1 foi alterada como mostrado na Equação 2.

$$
f(\mathbf{x})=\sum_{t=1}^{n}\left(r\left(a_{t}, \mathbf{s}_{t}, \mathbf{m}_{t}\right)+\left[\beta p\left(\mathbf{s}_{t}\right)+\sigma j(\theta)\right]\right)
$$

As diferenças em relação à Equação 1 ocorrem em $h\left(\mathbf{s}_{t}, \mathbf{m}_{t}\right)$, que assume valor 1 se a leitura atual dos sensores não se iguala à nenhuma das leituras armazenadas em $\mathbf{m}_{t}$. Caso contrário, $h\left(\mathbf{s}_{t}, \mathbf{m}_{t}\right)$ pode assumir dois valores: 1) $h\left(\mathbf{s}_{t}, \mathbf{m}_{t}\right)=0,2$ se o número de sensores ativos é zero, 2) $h\left(\mathbf{s}_{t}, \mathbf{m}_{t}\right)=0,1$ se pelo menos um dos sensores estiver ativo. Aqui, $j(\theta)$ é uma variável que assume valor -1 quando a soma dos ângulos em passos consecutivos é maior ou igual a $180^{\circ}$, i.e., $\theta \geq 180^{\circ}$; $\theta$ é soma dos ângulos em passos consecutivos e $\sigma$ é uma constante escolhida no intervalo $[0,5]$.

Para os experimentos com o fitness da Equação 2, foram mantidos os mesmos valores dos parâmetros da ESN. Para o AG, apenas os valores de $\beta$ e $\alpha$ foram modificados. Para equilibrar os termos de punição e recompensa, foi utilizado $\alpha=0,4, \gamma=3$ e $\beta$ entre 0,2 e 6 . Aqui, $\beta=0,2$ foi escolhido para simular o rato com menos ansiedade, semelhante a $\beta=0,5$ para os experimentos anteriores. Para simular o rato com mais 
ansiedade, $\beta=1,6$ foi escolhido, e corresponde a $\beta=6$ nos experimentos anteriores.

Na Figura 4 são apresentados as trajetórias e os respectivos mapas de calor de algumas simulações realizados com o tamanho de memória 20, com $\beta=0,2$ (Figura 4.a - 4.f) e $\beta=1,6$ (Figura 4.g - 4.1). Os pontos inicial e final de cada trajetória estão marcados em amarelo na forma de círculo e quadrado, respectivamente.

Os resultados quantitativos para esses experimentos podem ser vistos na Figura 5. Foram calculadas as médias de 30 execuções do AG com $\beta=0,2$ e $\beta=1,6$ para as métricas citadas anteriormente: 1) Média do número de entradas do rato virtual para as três regiões (Figura 5.a), 2) Média do tempo de permanência em cada uma das três regiões (Figura 5.b) e 3) Média da distância percorrida na região central (Figura 5.c). Para análise estatística utilizou-se o teste de Wilcoxon (signed ranks). As diferenças entre os grupos foram consideradas estatisticamente significantes para o nível de significância $p<0,001$.

(a)

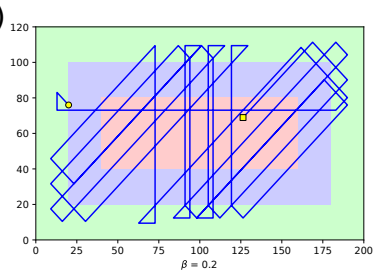

(d)

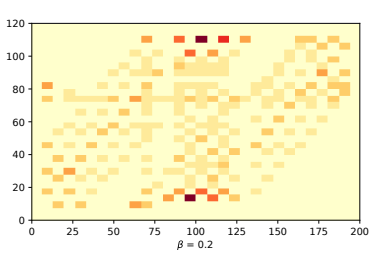

(g)

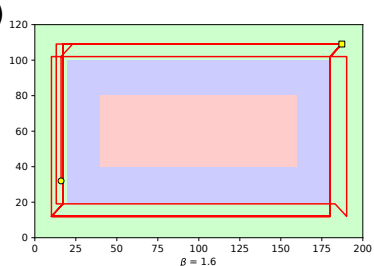

(j)

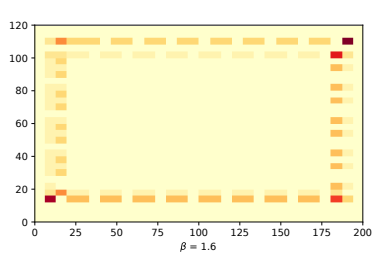

(b)

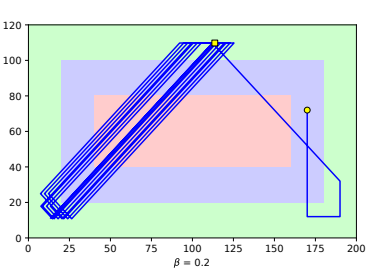

(e)

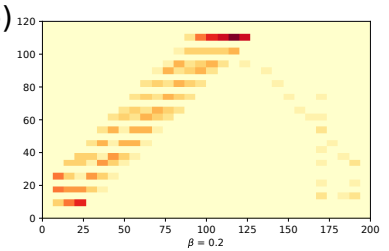

(h)

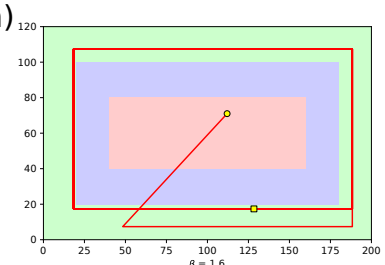

(k)

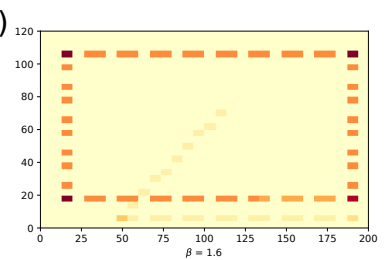

(c)

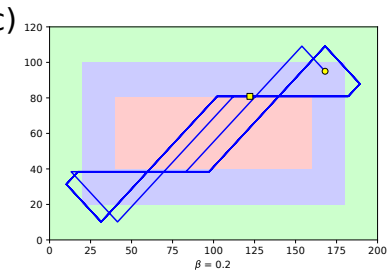

(f)

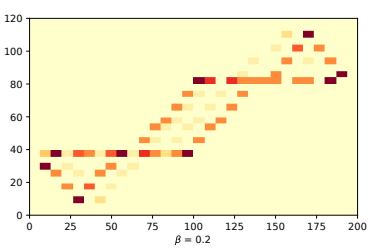

(i)

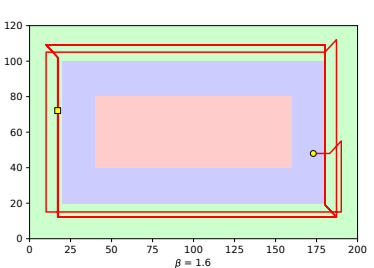

(I)

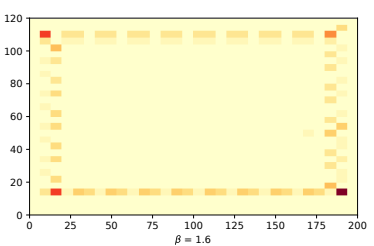

Figura 4. Trajetórias e mapas de calor da simulação para o teste do campo aberto com memória de tamanho 20 e $\beta=0,2$ ((a) - (f)) e $\beta=1,6$ ((g) - (l)).

É possível ver na Figura 4 que para $\beta=0,2$ o comportamento do rato na arena é mais exploratório e, para $\beta=1,6$ (maior punição), o rato tende a ficar mais tempo na região externa da arena, como mostrado nos mapas de calor.

Esses comportamentos podem ser melhor observados na Figura 5. Na Figura 5.a é possível observar que os ratos virtuais $\operatorname{com} \beta=0,2$ exploram mais a arena, tendo médias do número de entradas nas três regiões maiores do que os ratos virtuais com $\beta=1,6$. Eles apresentam média de tempo na região central (C) e intermediária (I) maior quando 
(a)

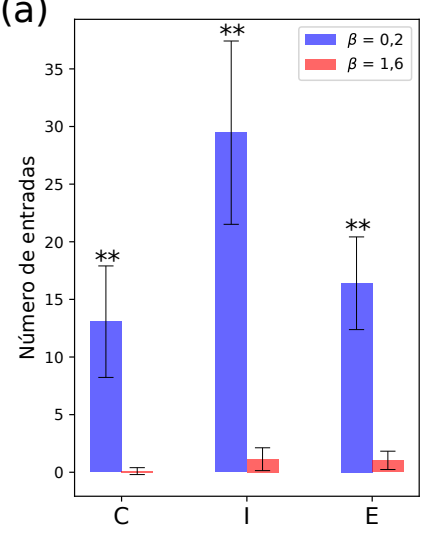

(b)

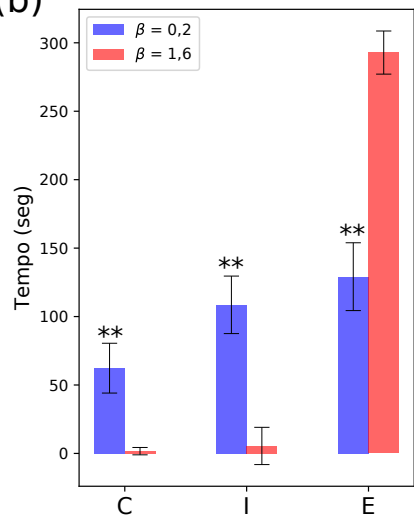

(c)

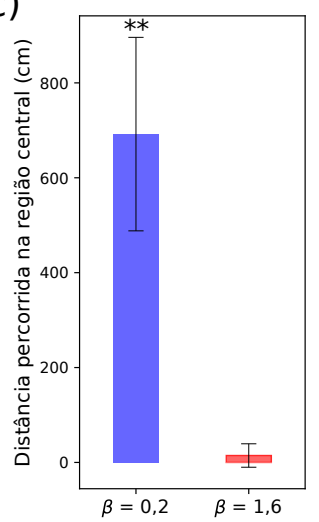

Figura 5. Resultados da simulação para o teste do campo aberto com memória 20 e $\beta=0,2$ (azul) e $\beta=1,6$ (vermelho). (a) Média do número de entradas do rato virtual para as três regiões (C, I, E), (b) Média do tempo de permanência em cada uma das três regiões (C, I, E) e (c) Média da distância percorrida na região central. $O$ símbolo $\left.{ }^{* *}\right)$ indica que os resultados para $\beta=0,2$ e $\beta=1,6$ são de populações diferentes de acordo com o teste de Wilcoxon (signed ranks) com $p<0,001$.

comparadas aos ratos virtuais $\operatorname{com} \beta=1,6$ (Figura 5.b). Estes, por sua vez, passam mais tempo perto da parede, tendo a média de tempo na região externa (E) muito maior (Figura 5.c) e média da distância percorrida na região central muito menor (Figura 5.c).

O efeito da variação de $\beta$ no comportamento do rato virtual pode ser observado na Figura 6, onde são mostradas em função de $\beta$ as médias de tempo e número de entradas em cada região. Em preto, encontram-se as curvas para $\beta$ com valores entre 0,2 e 1,6 (incrementos de 0,2 ). Os resultados para cada foram obtidos com a média de 30 execuções do AG. Em vermelho, encontram-se as curvas obtidas com o ajuste exponencial dos resultados de cada $\beta$. Assim, o parâmetro $\beta$ pode ser utilizado para reproduzir o efeito da dosagem de drogas que controlam a ansiedade em ratos no teste do campo aberto.

A média do número de entradas em cada região, assim como a média de tempo na região central e intermediária, decresce exponencialmente com o aumento do valor de $\beta$. Já a média de tempo na região externa cresce exponencialmente com o aumento do valor de $\beta$. Esses resultados são esperados, uma vez que o aumento do valor de está relacionado ao aumento da punição e, portanto, ao aumento do medo. Assim, quanto maior o , menor o caráter exploratório do rato virtual e maior o tempo gasto na região externa.

\subsection{Robô Evolutivo}

Os experimentos com o robô evolutivo foram realizados para simular o comportamento dos ratos em um ambiente real. Para simular as diferenças de comportamento, as melhores soluções obtidas nas simulações com a função de fitness da equação 1 foram testadas no robô real. Foram utilizados para o parâmetro $\beta$ os valores 0,5 e 6; 10 para a memória.

O ambiente real possui desafios como: diferenças na iluminação do ambiente, variações de atrito na superfície em que o robô se movimenta, precisão dos sensores e dos movimentos do robô. Para $\beta=0,5$, os indivíduos que exploram mais o ambiente foram testados no robô e os resultados obtidos foram semelhantes aos da simulação, ou seja, o robô explorou bem as três regiões do ambiente. Para $\beta=6$, os indivíduos testados no 
(a)

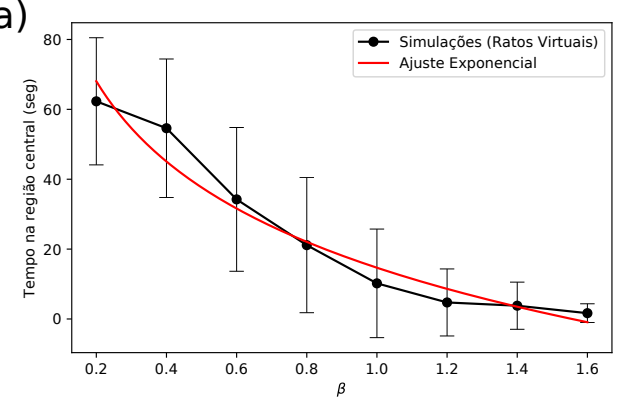

(c)

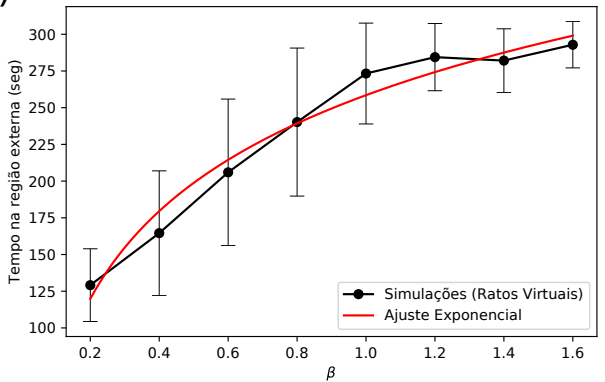

(e)

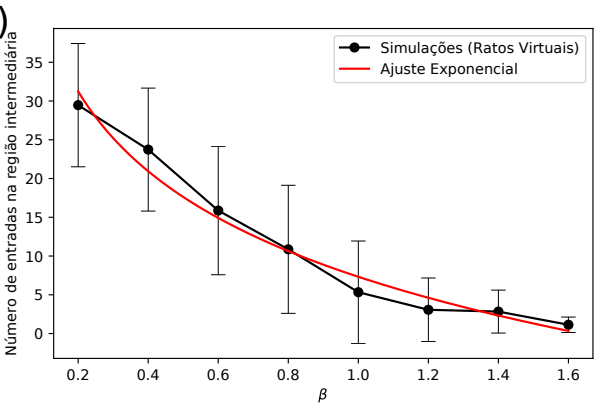

(b)

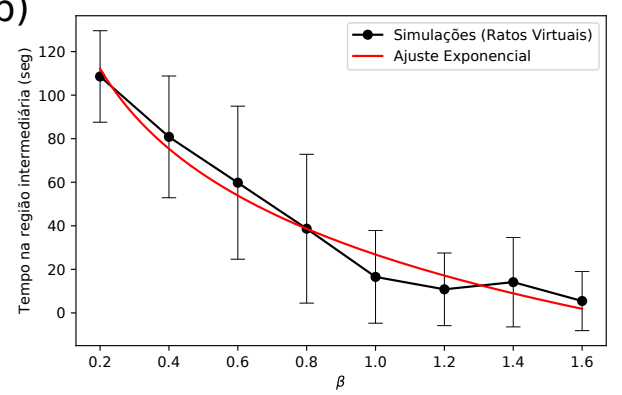

(d)

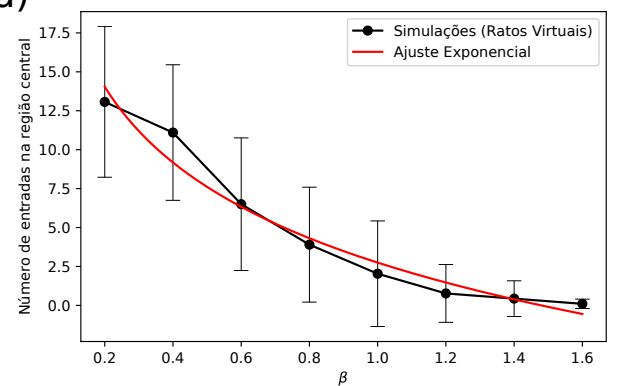

(f)

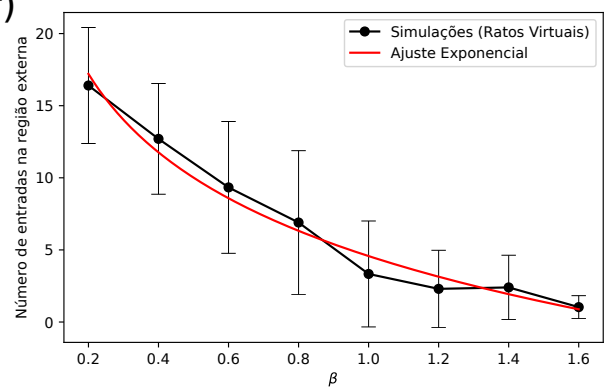

Figura 6. Média de tempo gasto na região (a) central, (b) intermediária e (c) externa, em relação a $\beta$. Média do número de entradas na região (d) central, (e) intermediária e (f) externa, em relação a $\beta$.

robô também foram semelhantes aos da simulação e o robô explorou menos o ambiente, evitando a região central. Os vídeos de dois desses experimentos estão disponíveis em:

- https://youtu.be/Dn6QEQj8vbU $(\beta=6)$

- https://youtu.be/IDWN8rla7sA $(\beta=0,5)$

\section{Conclusões}

De maneira geral, os resultados mostram que o rato virtual simula características relevantes do comportamento exploratório do rato no teste de campo aberto. Quando o nível de ansiedade é maior, os ratos virtuais evitam a região central do campo aberto, a parte mais exposta da arena. Um aumento de tempo na região central do campo aberto é interpretado como uma diminuição na ansiedade do rato. Tal comportamento pode ser observado nos resultados obtidos com ambas as funções de fitness. O modelo permite simular o aumento da ansiedade por meio de um parâmetro $(\beta)$ que controla o conflito entre medo e caráter exploratório. Os testes com o robô mostraram que, mesmo em uma situação desafiadora como o ambiente real, o modelo proposto simula o comportamento esperado. Assim, o modelo proposto pode ser um instrumento útil para o estudo de mecanismos de comportamento. Ratos virtuais derivados do modelo proposto podem, no futuro, permitir reduzir 
o número de experimentos com ratos reais e trazer um maior entendimento sobre aspectos relacionados à tomada de decisão dos agentes (ratos reais e virtuais).

\section{Agradecimentos}

Os autores agradecem à FAPESP (processo 2017/11139-0) pelo apoio financeiro.

\section{Referências}

Costa, A. A., Morato, S., Roque, A. C., and Tinós, R. (2014). A computational model for exploratory activity of rats with different anxiety levels in elevated plus-maze. Journal of neuroscience methods, 236:44-50.

Costa, A. d. A. (2015). Investigação de modelos comportamentais de ratos por meio de algoritmos genéticos. PhD thesis, Universidade de São Paulo.

de Andrade Costa, A. and Tinós, R. (2016). Investigation of rat exploratory behavior via evolving artificial neural networks. Journal of neuroscience methods, 270:102-110.

Giddings, J. M. (2002). Modeling the behavior of rats in an elevated plus-maze. $\mathrm{PhD}$ thesis, Acadia University.

Hogg, S. (1996). A review of the validity and variability of the elevated plus-maze as an animal model of anxiety. Pharmacology Biochemistry and Behavior, 54(1):21-30.

Jaeger, H. and Haas, H. (2004). Harnessing nonlinearity: Predicting chaotic systems and saving energy in wireless communication. science, 304(5667):78-80.

Miranda, D., Conde, C., Celis, C., and Corzo, S. (2009). Modelado del comportamiento de ratas en laberinto en cruz elevado basado en redes neuronales artificiales. Revista Colombiana de Física, 41(2):406.

Montgomery, K. (1955). The relation between fear induced by novel stimulation and exploratory drive. Journal of comparative and physiological psychology, 48(4):254.

Salum, C., Morato, S., and Roque-da Silva, A. C. (2000). Anxiety-like behavior in rats: a computational model. Neural networks, 13(1):21-29.

Seibenhener, M. L. and Wooten, M. C. (2015). Use of the open field maze to measure locomotor and anxiety-like behavior in mice. Journal of visualized experiments: JoVE, (96).

Shimo, H. K., Roque, A. C., Tinos, R., Tejada, J., and Morato, S. (2010). Use of evolutionary robots as an auxiliary tool for developing behavioral models of rats in an elevated plus-maze. In Neural Networks (SBRN), 2010 Eleventh Brazilian Symposium on, pages 217-222. IEEE.

Tejada, J., Bosco, G. G., Morato, S., and Roque, A. C. (2010). Characterization of the rat exploratory behavior in the elevated plus-maze with markov chains. Journal of neuroscience methods, 193(2):288-295.

Walsh, R. N. and Cummins, R. A. (1976). The open-field test: a critical review. Psychological bulletin, 83(3):482.

Webb, B. (2001). Can robots make good models of biological behaviour? Behavioral and brain sciences, 24(6):1033-1050. 